Research article

\title{
Whither Polyalthia (Annonaceae) in Peninsular Malaysia? Synopses of Huberantha, Maasia, Monoon and Polyalthia s.s.
}

\author{
Ian M. TURNER * \& Timothy M.A. UTTERIDGE \\ Royal Botanic Gardens Kew, Richmond, Surrey, TW9 3AE, United Kingdom \\ * Corresponding author: turner187@btinternet.com
}

\begin{abstract}
An updated classification of Polyalthia in Peninsular Malaysia is presented. A synopsis (listing of species with synonymy and typification, and keys to species) is presented for the genera Huberantha, Maasia, Monoon and Polyalthia sensu stricto. One new species (Polyalthia pakdin I.M.Turner \& Utteridge sp. nov.) is described and a conservation assessment presented for it. Monoon xanthopetalum Merr. represents a new record for Peninsular Malaysia. Six new lectotypes are designated.
\end{abstract}

Keywords. Enicosanthum, Huberantha, Maasia, Monoon, Polyalthia.

Turner I.M. \& Utteridge T.M.A. 2016. Whither Polyalthia (Annonaceae) in Peninsular Malaysia? Synopses of Huberantha, Maasia, Monoon and Polyalthia s.s. European Journal of Taxonomy 183: 1-26. http://dx.doi. org/10.5852/ejt.2016.183

\section{Introduction}

When Sinclair (1955) revised the Annonaceae of the Malay Peninsula he recognised 32 species of Polyalthia Blume (including Polyalthia evecta Finet \& Gagnep. from Peninsular Thailand still unrecorded from Peninsular Malaysia, and the cultivated Polyalthia longifolia (Sonn.) Thwaites). The 30 native species made Polyalthia the largest genus in the family as represented in the Malayan flora. The genus was characterised by Sinclair largely in terms of floral morphology including subequal corolla whorls of spreading, relatively flat, petals, numerous flat-topped stamens and many carpels with $1-5$ ovules each. Sinclair considered the genus to be divisible into two sections that he called 'Polyalthia section Eu-Polyalthia' (correctly Polyalthia section Polyalthia) and Polyalthia section Monoon (Miq.) Benth. \& Hook.f. Sinclair appeared confident in the classification, noting the Polyalthia was a large genus 'but very uniform'. However, in recent years the homogeneity of Polyalthia has come under close scrutiny with a growing conviction that it represented an unnatural grouping. Molecular analyses have confirmed the polyphyletic nature of Polyalthia sensu lato leading to a process of dismemberment as species have been transferred to various different genera. Of relevance to the species included in Polyalthia by Sinclair, section Monoon has been raised again to generic rank (Xue et al. 2012) (14 spp. of Sinclair's native Polyalthia species) and includes Enicosanthum Becc. (7 spp. recognised by Sinclair). Three of Sinclair's section Monoon species are now recognised in the new genus Maasia Mols et al. (Mols et al. 2008) and two in Huberantha Chaowasku in Chaowasku et al. [Chaowasku et al. 2015, originally named Hubera Chaowasku in Chaowasku et al. (Chaowasku et al. 2012) but this was considered to be an illegitimate later homonym of Huberia DC. (Melastomataceae)]. The 17 species Sinclair recognised in 'section Eu-Polyalthia' are all now deemed to belong in Polyalthia sensu stricto. 
Some Asian Polyalthia species have also been transferred to Marsypopetalum Scheff. (Xue et al. 2011), but this has not affected any of the species native to Peninsular Malaysia.

In this paper, we review these changes in the taxonomy of Polyalthia and associated genera in Peninsular Malaysia. This is done by providing synopses of the four genera Huberantha, Maasia, Monoon Miq. and Polyalthia including keys to the species. One new species of Polyalthia is described and a conservation assessment for it provided.

\section{Materials and methods}

Material in the herbarium of the Royal Botanic Gardens Kew $(\mathrm{K})$ and a loan of Annonaceae specimens from the herbarium of the Forest Research Insitute of Malaysia (KEP) was examined. For the new species, specimens were geo-referenced using Hamidah et al. (2011), and a conservation assessment was made following IUCN criteria (IUCN 2012) using Geocat to estimate the Extent of Occurrence (EOO) and Area of Occupancy (AOO) (http://geocat.kew.org; Bachman et al. 2011).

\section{Results}

Order Magnoliales Juss. ex Bercht. \& J.Presl

Family Annonaceae Juss.

Subfamily Malmeoideae Chatrou \& al.

Tribe Miliuseae Hook.f. \& Thomson

\section{Key to genera}

1. Lower surface of leaves densely covered with minute papillae giving a white or glaucous appearance

- Leaves not white or glaucous below

2. Leaf venation herringbone (eucamptodromous) with insertion of lateral veins to the midrib decurrent; intercostal venation scalariform to subscalariform

- Leaf venation looping (brochidodromous) with insertion of the lateral veins to the midrib not decurrent; intercostal venation reticulate 3

3. Leaf base generally more or less symmetrical, not (sub)cordate. Ovules 1 per carpel. Seeds 1 per monocarp

- Leaf base generally more or less asymmetrical, often (sub)cordate. Ovules usually 2-6 per carpel. Seeds usually 2 or more per monocarp

Polyalthia

\section{Maasia Mols et al.}

Systematic Botany 33: 493 (Mols et al. 2008). - Type: Maasia hypoleuca (Hook.f. \& Thomson) Mols et al.

\section{Key to species of Maasia}

1. Leaves generally not exceeding $8 \mathrm{~cm}$ long, $3 \mathrm{~cm}$ wide. Flowering pedicel to $5 \mathrm{~mm}$ long, petals to $6 \mathrm{~mm}$ long. Monocarps ellipsoidal, few ( 5 or less), not exceeding $2 \mathrm{~cm}$ long .......

- Leaves generally more than $8 \mathrm{~cm}$ long, $3 \mathrm{~cm}$ wide. Flowering pedicel more then $5 \mathrm{~mm}$ long, petals more than $6 \mathrm{~mm}$ long. Monocarps globose or if ellipsoidal then many (generally 10 or more) and more then $2 \mathrm{~cm}$ long at maturity 
TURNER I.M. \& UTTERIDGE T.M.A., Whither Polyalthia (Annonaceae) in Peninsular Malaysia?

2. Flowering pedicel more than $17 \mathrm{~mm}$ long, c. $1 \mathrm{~mm}$ thick, petals more than $1 \mathrm{~cm}$ long. Monocarps ellipsoidal..... M. sumatrana

- Flowering pedicel to $17 \mathrm{~mm}$ long, c. $0.5 \mathrm{~mm}$ thick, petals to $8 \mathrm{~mm}$ long. Monocarps globose

M. glauca

\section{Maasia glauca (Hassk.) Mols et al.}

Systematic Botany 33: 493 (Mols et al. 2008). - Uvaria glauca Hassk., Flora (Beiblätter) 25, 2 (2): 31 (Hasskarl 1842). - Guatteria glauca (Hassk.) Miq., Flora van Nederlandsch Indië 1 (2): 49 (Miquel 1858), nom. illegit., non G. glauca Ruiz \& Pav. (Ruiz \& Pavon 1798). - Monoon glaucum (Hassk.) Miq., Annales Musei Botanici Lugdunu-Batavi 2: 19 (Miquel 1865). - Polyalthia glauca (Hassk.) F.Muell., Descriptive notes on Papuan plants. Appendix: 95 (von Mueller 1877). - Lectotype (designated by Turner 2011: 46): Indonesia, Java, J.K. Hasskarl s.n. (L (L 0188666)). - Epitype (designated by Turner 2011: 46): Indonesia, Java: Udjung Kulon Reserve, Mt Pajung, 17 Nov. 1960, A.J.G.H. Kostermans et al. UNESCO 165 (holo-: L (L 0188665); iso-: A, BO, G, K).

Guatteria hypoleuca Miq., Flora van Nederlandsch Indië. Supplementum primum. Prodromus florae sumatranae: 381 (Miquel 1861). - Lectotype (designated by Rogstad 1989: 209): Indonesia, Sumatra: Priaman, s. dat., H. Diepenhorst s.n. [Herb. Bogor. no. 2095] (U (U 0000368)).

Unona merrittii Merr., Philippine Journal of Science 1, supplement III: 190 (Merrill 1906). — Polyalthia merrittii (Merr.) Merr., Philippine Journal of Science. Section C, Botany 10: 250 (Merrill 1915). Lectotype (designated by Turner 2011: 46): Philippines, Mindoro, Bongabong River, Feb. 1906, H.N. Whitford 1447 (hololecto-: K (K000691629); isolecto-: US).

Polyalthia parkinsonii Hutch., Bulletin of Miscellaneous Information, Kew 1917: 25 (Hutchinson 1917). - Lectotype (designated by Turner 2011: 46): India, Andaman Islands, Bom-Ling-La, 11 Feb. 1916, C.E. Parkinson 943 (hololecto-: K (K000691470); isolecto-: K (K000691469)).

\section{Description and distribution}

Sinclair (1955: 321), Kochummen (1972: 88), Rogstad (1989: 209-212), Turner (2014: 74-75).

Maasia hypoleuca (Hook.f. \& Thomson) Mols et al.

Systematic Botany 33: 493 (Mols et al. 2008). - Polyalthia hypoleuca Hook.f. \& Thomson, The Flora of British India 1: 63 (Hooker \& Thomson 1872). - Lectotype (designated by Rogstad 1989: 227): Singapore, Sep. 1867, A.C. Maingay 1516 A [Kew Distrib. no. 50] (K (K000691444)).

\section{Description and distribution}

Sinclair (1955: 320-321), Kochummen (1972: 88), Rogstad (1989: 227-229), Turner (2014: 75-76).

\section{Maasia sumatrana (Miq.) Mols et al.}

Systematic Botany 33: 493 (Mols et al. 2008). - Guatteria sumatrana Miq., Flora van Nederlandsch Indië. Supplementum primum. Prodromus florae sumatranae: 380 (Miquel 1861). - Monoon sumatranum (Miq.) Miq., Annales Musei Botanici Lugdunu-Batavi 2: 19 (Miquel 1865). - Polyalthia sumatrana (Miq.) Kurz, Journal of the Asiatic Society of Bengal. Part II (Natural History \&c.) 43: 53 (Kurz 1874). - Lectotype (designated by Rogstad 1989: 220): Indonesia, Sumatra, Priaman Province, H. Diepenhorst s.n. [Herb. Bogor. 2342] (U (U 0000372)).

\section{Description and distribution}

Sinclair (1955: 319-320), Kochummen (1972: 91), Rogstad (1989: 229-221), Turner (2014: 76-78). 
Huberantha Chaowasku in Chaowasku et al.

Kew Bulletin 70 (2)-23: 1 (Chaowasku et al. 2015). - Hubera Chaowasku in Chaowasku et al., Phytotaxa 69: 46 (Chaowasku et al. 2012), non Huberia DC. (de Candolle 1828). - Type: Hubera cerasoides (Roxb.) Chaowasku.

\section{Key to species of Huberantha}

1. Sepals suborbicular, to $3 \mathrm{~mm}$ long; petals drying uniformly pale brown

H. jenkinsii

- Sepals triangular, c. $6 \mathrm{~mm}$ long or more; petals often drying with powdery yellow patches

H. rumphii

Huberantha jenkinsii (Hook.f. \& Thomson) Chaowasku in Chaowasku et al.

Kew Bulletin 70 (2)-23: 2 (Chaowasku et al. 2015). - Guatteria jenkinsii Hook.f. \& Thomson, Flora Indica: 141 (Hooker \& Thomson 1855). - Polyalthia jenkinsii (Hook.f. \& Thomson) Hook.f. \& Thomson, The Flora of British India 1: 64. (Hooker \& Thomson 1872). - Hubera jenkinsii (Hook.f. \& Thomson) Chaowasku in Chaowasku et al., Phytotaxa 69: 48 (Chaowasku et al. 2012). - Lectotype (designated by Turner 2011: 73): India, Assam, s.dat., F. Jenkins s.n. (K (barcode no. K000691481)).

Polyalthia andamanica Kurz ex King, Journal of the Asiatic Society of Bengal. Part II (Natural History \&c.) 61: 53 (King 1892). - Lectotype (designated by Turner 2011: 74): India, Andaman Islands, South Andaman, Namanagha, 13 Dec. 1890, King's Collector [H.H. Kunstler] s.n. (hololecto-: K (K000739193); isolecto-: K).

Polyalthia havilandii Boerl., Icones Bogorienses 1: 107, t. 66 (Boerlage 1899a). - Type: Borneo, Sarawak, near Kuching, 15 Feb. 1894, G.D. Haviland 2 (holo-: BO (sheet no. BO-1352593); iso-: L (L 0188829)).

Polyalthia cumingiana Merr., Publications of the Bureau of Science Government Laboratories 35: 71 (Merrill 1905). - Lectotype (designated by Turner 2011: 74): Philippines, Luzon, Prov. Tayabas, H. Cuming 827 (hololecto-: K (K000691639); isolecto-: K [×2], NY, WU).

Unona agusanensis Elmer, Leaflets of Philippine Botany 5: 1743 (Elmer 1913). - Polyalthia agusanensis (Elmer) Merr., Philippine Journal of Science. Section C, Botany 10: 250 (Merrill 1915). - Lectotype (designated by Turner 2011: 74): Philippines, Mindanao, Province of Agusan, Cabadbaran (Mt Urdaneta), Sep. 1912, A.D.E. Elmer 13654 (hololecto:- NY (00026313); isolecto-: A, K, L).

\section{Description and distribution}

Sinclair (1955: 305-306), Kochummen (1972: 88), Turner (2014: 71-72).

Huberantha rumphii (Blume ex Hensch.) Chaowasku in Chaowasku et al.

Kew Bulletin 70 (2)-23: 2 (Chaowasku et al. 2015). - Guatteria rumphii Blume ex Hensch., Vita G. E. Rumphii: 153 (Henschel 1833). - Polyalthia rumphii (Blume ex Hensch.) Merr., An Enumeration of Philippine flowering plants 2: 162 (Merrill 1923). - Hubera rumphii (Blume ex Hensch.) Chaowasku in Chaowasku et al., Phytotaxa 69: 50 (Chaowasku et al. 2012). - Type: Entirely based on Arbor nigra parvifolia of Rumphius, Herbarium Amboinense 3: 10, t 4, f 2, t 5 (Rumphius 1743).

Guatteria canangioides Rchb.f. \& Zoll. in Zollinger, Linnaea 29: 322-323 (Zollinger 1858). - Monoon canangioides (Rchb.f. \& Zoll.) Miq., Annales Musei Botanici Lugdunu-Batavi 2: 18 (Miquel 1865). Polyalthia canangioides (Rchb.f. \& Zoll.) Boerl., Catalogus Plantarum Phanerogamarum quae in Horto Botanico Bogoriensi Coluntur Herbaceis Exceptis (1): 19 (Boerlage 1899b). - Lectotype (designated by Turner 2011: 78): Indonesia, Sumatra, Province of Lampong, H. Zollinger 3047 (hololecto-: P (P01983362); isolecto-: ?BM, ?P). 
Guatteria parveana Miq., Flora van Nederlandsch Indië 1 (2): 48 (Miquel 1858). - Polyalthia canangioides var. parveana (Miq.) Boerl., Catalogus Plantarum Phanerogamarum quae in Horto Botanico Bogoriensi Coluntur Herbaceis Exceptis (1): 20 (Boerlage 1899b). Type: Based on manuscript description by $\mathrm{H}$. Zollinger of Uvaria parveana .

Unona borneensis Miq., Annales Musei Botanici Lugdunu-Batavi 2: 11 (Miquel 1865). - Desmos borneensis (Miq.) Merr. Journal of the Straits Branch of the Royal Asiatic Society, Special Number: 255 (Merrill 1921). - Lectotype (designated by Turner 2011: 78): Borneo, Poeloe Lampei, P.W. Korthals s.n. (L (L 0189189)).

Polyalthia scortechinii King, Journal of the Asiatic Society of Bengal. Part II (Natural History \&c.) 61: 56 (King 1892). - Lectotype (designated by Turner 2011: 78): Peninsular Malaysia, Perak, Larut, May 1884, King's Collector [H.H. Kunstler] 6125 (hololecto-: K (K000739187); isolecto-: CAL, DD, K (K000739186)).

Polyalthia kunstleri King, Journal of the Asiatic Society of Bengal. Part II (Natural History \&c.) 61: 55 (King 1892). - Lectotype (designated by Turner 2011: 78): Peninsular Malaysia, Perak, Larut, Jan. 1883, King's Collector [H.H. Kunstler] 3767 (hololecto-: K (K000691450); isolecto-: CAL, DD, K (K000691451), MEL).

Polyalthia glandulosa Merr., Philippine Journal of Science. Section C, Botany 10: 247 (Merrill 1915). - Lectotype (designated by Turner 2011: 78): Philippines, Mindanao, District of Zamboanga, Santa Maria, near Mount Pulangbato, 4 Oct. 1912, J. Reillo Bur. Sci. 16460 (hololecto-: US (US00098663); isolecto-: K (K000691642), L, P (P00372685)).

\section{Description and distribution}

Sinclair (1955: 306-307), Kochummen (1972: 89) Turner (2014: 72).

\section{Monoon Miq.}

Annales Musei Botanici Lugdunu-Batavi 2: 15 (Miquel 1865). - Lectotype (designated by Saunders \& Xue 2011: 236): Monoon lateriflorum (Blume) Miq.

Enicosanthum Becc., Nuovo Giornale Botanico Italiano 3: 183 (Beccari 1871). - Type: Enicosanthum paradoxum Becc.

Marcuccia Becc., Nuovo Giornale Botanico Italiano 3: 181, t. 3 (Beccari 1871). - Type: Marcuccia grandiflora Becc.

Griffithia Maingay ex King, Annals of the Royal Botanic Garden (Calcutta) 4: 8 (King 1893), non Griffithia Wight \& Arn. (Wight \& Arnott 1834). - Griffithianthus Merr., Philippine Journal of Science. Section C, Botany 10: 231 (Merrill 1915). - Lectotype (designated by Merrill 1915: 231): Griffithia magnoliipetala Maingay ex King.

Woodiella Merr., Journal of the Straits Branch of the Royal Asiatic Society 85: 187 (Merrill 1922), non Woodiella Sacc. \& P.Syd. (Sydow 1899). - Woodiellantha Rauschert, Taxon 31: 555 (Rauschert 1982). - Type: Woodiella sympetala Merr.

Cleistopetalum H.Okada, Acta Phytotaxonomica et Geobotanica 47: 4 (Okada 1996). - Type: Cleistopetalum borneense H.Okada.

\section{Key to species of Monoon}

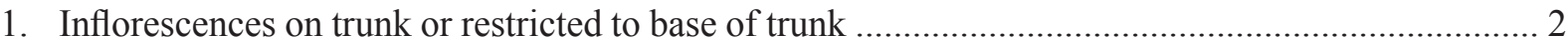

- Inflorescences in leaf axils, or on twigs behind leaves (sometimes on branches) .............................. 6

2. Inflorescences borne on slender, flexible, branched basal flagellae M. hypogaeum

- Not so 3

3. Inflorescences on stout, sparsely branching axes to at least $7 \mathrm{~cm}$ long M. praestigiosum 
- Inflorescences on woody tubercles or much-branched axes less than $7 \mathrm{~cm}$ long.....

4. Inflorescences on woody tubercles

M. anomalum

- Inflorescences on much-branched axes

5. Leaves with $8-10$ pairs of lateral nerves. Outer petals to $2.5 \mathrm{~cm}$ long, $0.5 \mathrm{~cm}$ wide. Monocarps with blunt beak . . .

M. borneense

- Leaves with c. 20 pairs of lateral nerves. Outer petal to $7 \mathrm{~cm}$ long, $2.5 \mathrm{~cm}$ wide. Monocarps with hard, sharp beak

M. congregatum

6. Leaves membranous. Inflorescences axillary, few-flowered (generally more than one). Sepals more than $8 \mathrm{~mm}$ long. Monocarps globose to ellipsoidal with rounded to slightly blunt apex

M. membranifolium

- Leaves chartaceous to coriaceous. Inflorescences single to multi-flowered but if few-flowered and axillary then with sepals to $6 \mathrm{~mm}$ long. Monocarps ellipsoidal with blunt to beaked apex .............. 7

7. Young flowers with conical 'bud' stage; sepals more than $6 \mathrm{~mm}$ wide ........................................ 8

- Young flowers not with a conical 'bud' stage; sepals less than $6 \mathrm{~mm}$ wide .................................... 11

8. Leaves with generally more than 20 pairs of lateral nerves. Flower pedicels more than $2.5 \mathrm{~cm}$ long .... M. macranthum

- Leaves with up to 20 pairs of lateral nerves. Flower pedicels to $1 \mathrm{~cm}$ long 9

9. Twigs densely tomentose. Leaves with dense hairs on nerves below. Flower pedicel to $12 \mathrm{~mm}$ long ... M. fuscum

- Twigs glabrescent. Leaves glabrous to glabrescent beneath. Flower pedicel generally more than $12 \mathrm{~mm}$ long

10. Leaves to $17 \mathrm{~cm}$ long, $7 \mathrm{~cm}$ wide. Sepals much broader than long. Monocarps woolly hairy, apex rounded

M. cupulare

- Leaves generally more than $17 \mathrm{~cm}$ long, $7 \mathrm{~cm}$ wide. Sepals about as long as broad. Monocarps not woolly hairy, apex beaked

M. magnoliiflorum

11. Petals distinctly longitudinally nerved. Monocarps subsessile, pale tomentose M. pachyphyllum

- Petals not longitudinally nerved. Monocarps stipitate, glabrous to tomentose

12 (Leaves more than $24 \mathrm{~cm}$ long, $7 \mathrm{~cm}$ wide). Flowers borne distichously on sparsely branched hairy axes to $2 \mathrm{~cm}$ long, $4 \mathrm{~mm}$ thick. Petals thick and fleshy. Monocarps with stipe to $14 \mathrm{~mm}$ long, shorter than seed-bearing portion

M. malayanum

- (Leaves generally smaller). Flowers borne in fascicles or on very short and relatively slender axes. Petals relatively thin. Monocarps with stipe generally exceeding length of seed-bearing portion ... 13

13. Flowering pedicel c. $0.5 \mathrm{~mm}$ thick; sepals to $2 \mathrm{~mm}$ long; petals to $3 \mathrm{~mm}$ wide M. asteriellum

- Flowering pedicel at least $1 \mathrm{~mm}$ thick; sepals more than $2 \mathrm{~mm}$ long; petals $4 \mathrm{~mm}$ wide or more ... 14

14. Petals 4-5 mm wide. Monocarps densely short brown tomentose, stipe attachment eccentric.

M. xanthopetalum

- Petals $8 \mathrm{~mm}$ or more wide. Monocarps more or less glabrous, stipe attachment not eccentric ........ 15

15. Outer petals obovate (widest above midway), claw and blade distinct 16 
TURNER I.M. \& UTTERIDGE T.M.A., Whither Polyalthia (Annonaceae) in Peninsular Malaysia?

- Outer petals lanceolate to oblong-linear (widest at or below midway), little distinction between claw and blade 17

16. Leaves obovate, reticulations obscure from above M. hookerianum

- Leaves elliptic, reticulations distinct from above M. glabrum

17. Leaves generally less than $20 \mathrm{~cm}$ long and $5 \mathrm{~cm}$ wide; lateral nerves $9-13$ pairs. Sepals $3-4 \mathrm{~mm}$ long .. M. sclerophyllum

- Leaves generally more than $20 \mathrm{~cm}$ long and $5 \mathrm{~cm}$ wide; lateral nerves $14-15$ pairs. Sepals to $3 \mathrm{~mm}$ long.

M. lateriflorum

\section{Monoon anomalum (Becc.) B.Xue \& R.M.K.Saunders}

Taxon 61: 1030 (Xue et al. 2012). - Polyalthia anomala Becc., Nuovo Giornale Botanico Italiano 3: 188 (Beccari 1871). - Type: Borneo, Sarawak, Mt Mattan, May 1866, O. Beccari P.B. 1605 (holo-: FI-B (Erb. Becc. no. 481)).

Polyalthia clavigera King, Journal of the Asiatic Society of Bengal. Part II (Natural History \&c.) 61: 60 (King 1892). - Lectotype (designated by Turner 2010a): Peninsular Malaysia, Perak, Waterfall Hill, Jun. 1888, L. Wray 2075 (hololecto-: K (K000691553); isolecto-: CAL, SING).

Polyalthia glomerata King, Journal of the Asiatic Society of Bengal. Part II (Natural History \& c.) 61: 61 (King 1892). - Lectotype (designated by Sinclair 1955: 318): Peninsular Malaysia, Perak, Jan. 1883, King's Collector [H.H. Kunstler] 3817 (hololecto-: K (K000691552); isolecto-: BM, L, P).

Polyalthia sumatrana var. macrocarpa Kochummen \& Whitmore in Kochummen et al., Federation Museums Journal 13: 134 (Kochummen et al. 1970). - Lectotype (designated here): Peninsular Malaysia, Johore, Labis, Compartment 81, 15 Apr 1967, T. Suppiah KEP 104976 (hololecto-: KEP (fruiting material, explicitly excluding the foliage material); isolecto-: $\mathrm{K}$ ).

Cleistopetalum sumatranum H.Okada, Acta Phytotaxonomica et Geobotanica 47: 5, Fig. 4 (Okada 1996). - Type: Indonesia, Sumatra, West Sumatra, Gunung Gadut, Bukit Pinang Pinang, c. $20 \mathrm{~km}$ east of Padang, 14 Dec. 1987, H. Okada 4607 (holo-: BO; iso-: KYO).

\section{Description and distribution}

Sinclair (1955: 318-319), Turner (2014: 110).

\section{Remarks}

Note that Polyalthia sumatrana var. macrocarpa was described from a mixed collection. Fruiting material of Monoon anomalum was confused with foliage of Maasia sumatrana. As the diagnosis focused on the characters of the fruits, we here designate the fruiting material as lectotype and exclude the foliage material.

Monoon asteriellum (Ridl.) B.Xue \& R.M.K.Saunders

Taxon 61: 1030 (Xue et al. 2012). - Polyalthia asteriella Ridl., Journal of the Straits Branch of the Royal Asiatic Society 82: 169 (Ridley 1920). Lectotype (designated here): Peninsular Malaysia, Perak, Larut Hills, 1892, H.N. Ridley 2986 (hololecto-: K (K000691443); isolecto-: CAL, SING (SING 0048679)).

\section{Description and distribution}

Sinclair (1955: 309-310). 
Monoon borneense (H. Okada) B.Xue \& R.M.K.Saunders

Taxon 61: 1030 (Xue et al. 2012). - Cleistopetalum borneense H.Okada, Acta Phytotaxonomica et Geobotanica 47: 4, Fig. 3 (Okada 1996). - Polyalthia sinclairiana I.M.Turner, Gardens' Bulletin, Singapore 58: 275 (Turner 2007). - Type: Borneo, East Kalimantan, Sungai Menubar, 12 Dec. 1980, Kato \& Wiriadinata 7119 (holo-: KYO; iso-: BO, L).

Polyalthia macropoda King, Journal of the Asiatic Society of Bengal. Part II (Natural History \&c.) 61: 60 (King 1892), non P. macropoda (Miq.) F.Muell. (von Mueller 1877). - Lectotype (designated by Turner 2007): Peninsular Malaysia, Perak, Jun. 1883, King's Collector [H.H. Kunstler] 4279 (hololecto-: K (K000691557); isolecto-: BM, L, P).

\section{Description and distribution}

Sinclair (1955: 316-317).

Monoon congregatum (King) B.Xue \& R.M.K.Saunders

Taxon 61: 1031 (Xue et al. 2012). - Polyalthia congregata King, Journal of the Asiatic Society of Bengal. Part II (Natural History \&c.) 61: 61 (King 1892). - Enicosanthum congregatum (King) Airy Shaw, Bulletin of Miscellaneous Information, Kew 1939: 277 (Airy-Shaw 1939). - Lectotype (designated by Xue et al. 2012: 1031): Peninsular Malaysia, Perak, Gopeng, Aug. 1883, King's Collector [H.H. Kunstler] 4831 (hololecto-: K (K000691419); isolecto-: CAL, DD, SING (SING0059443)).

\section{Description and distribution}

Sinclair (1955: 194).

Monoon cupulare (King) B.Xue \& R.M.K.Saunders

Taxon 61: 1031 (Xue et al. 2012). - Griffithia cupularis King, Annals of the Royal Botanic Garden (Calcutta) 4: 9, t. 219 (King 1893). - Griffithianthus cupularis (Maingay ex Hook.f. \& Thomson) Merr., Philippine Journal of Science. Section C, Botany 10: 231 (Merrill 1915). - Enicosanthum cupulare (King) Airy Shaw, Bulletin of Miscellaneous Information, Kew 1939: 277 (Airy-Shaw 1939). - Lectotype (designated by Xue et al. 2012: 1031): Peninsular Malaysia, Perak, Larut, May 1885, King 's Collector [H.H. Kunstler] 7630 (hololecto-: K (K000691422); isolecto-: BM (BM001050387), CAL).

\section{Description and distribution}

Sinclair (1955: 188-189).

Monoon fuscum (King) B.Xue \& R.M.K.Saunders

Taxon 61: 1031 (Xue et al. 2012). - Griffithia fusca King, Annals of the Royal Botanic Garden (Calcutta) 4: 10, t. 220 (King 1893). - Griffithianthus fuscus (Maingay ex Hook.f. \& Thomson) Merr., Philippine Journal of Science. Section C, Botany 10: 231 (Merrill 1915). Enicosanthum fuscum (King) Airy Shaw, Bulletin of Miscellaneous Information, Kew 1939: 277 (Airy-Shaw 1939). - Lectotype (designated by Xue et al. 2012: 1031): Peninsular Malaysia, Perak, Ulu Bubong, Jul. 1886, King's Collector [H.H. Kunstler] 10404 (hololecto-: K (K000691418); isolecto-: BM (BM001100029), CAL).

\section{Description and distribution}

Sinclair (1955: 189-190). 
TURNER I.M. \& UTTERIDGE T.M.A., Whither Polyalthia (Annonaceae) in Peninsular Malaysia?

Monoon glabrum (Hook.f. \& Thomson) B.Xue \& R.M.K.Saunders

Taxon 61: 1031 (Xue et al. 2012). - Ellipeia glabra Hook.f. \& Thomson, The Flora of British India 1: 52 (Hooker \& Thomson 1872). - Polyalthia glabra (Hook.f. \& Thomson) J.Sinclair, Gardens' Bulletin, Singapore 14: 315 (Sinclair 1955). - Type: Peninsular Malaysia, Malacca, 14 Dec. 1867, A.C. Maingay 3007 [Kew distribution no. 66] (holo-: K [×2] (K000691571, K000691570); iso-: CAL).

Polyalthia curtisii Ridl., Journal of the Straits Branch of the Royal Asiatic Society 54: 11 (Ridley 1910). - Lectotype (designated here): Peninsular Malaysia, Penang, Telok Bahang, Jun. 1901, C. Curtis 3644 (hololecto-: K (K000691572); isolecto-: SING).

\section{Description and distribution}

Sinclair (1955: 315).

Monoon hookerianum (King) B.Xue \& R.M.K.Saunders

Taxon 61: 1032 (Xue et al. 2012). - Polyalthia hookeriana King, Journal of the Asiatic Society of Bengal. Part II (Natural History \&c.) 61: 57 (King 1892). - Lectotype (designated by Turner 2011: 72): Peninsular Malaysia, Perak, Feb. 1884, King's Collector [H.H. Kunstler] 5550 (hololecto-: K (K000691563); isolecto-: CAL, P (P00601064)).

\section{Description and distribution}

Sinclair (1955: 312-315), Turner (2014: 113-115).

Monoon hypogaeum (King) B.Xue \& R.M.K.Saunders

Taxon 61: 1032 (Xue et al. 2012). - Polyalthia hypogaea King, Journal of the Asiatic Society of Bengal. Part II (Natural History \&c.) 61: 62 (King 1892). - Lectotype (designated by Turner 2011: 72): Peninsular Malaysia, Perak, Larut, 1881, King's Collector [H.H. Kunstler] 2437 (hololecto-: K (K000691555); isolecto-: CAL).

\section{Description and distribution}

Sinclair (1955: 315-316), Turner (2014: 115-116).

\section{Monoon lateriflorum (Blume) Miq.}

Annales Musei Botanici Lugdunu-Batavi 2: 19 (Miquel 1865). - Guatteria lateriflora Blume, Bijdragen tot de flora van Nederlandsch Indië: 20 (Blume 1825). - Lectotype (designated by Turner 2011: 74): Indonesia, Java: s.dat., Anon. s.n. (L (L 0188880)).

\section{Description and distribution}

Sinclair (1955: 310-311), Kochummen (1972: 88-89), Turner (2014: 116).

Monoon macranthum (King) B.Xue \& R.M.K.Saunders

Taxon 61: 1032 (Xue et al. 2012). - Polyalthia macrantha King, Journal of the Asiatic Society of Bengal. Part II (Natural History \& c.) 61: 54 (King 1892). - Enicosanthum macranthum (King) J.Sinclair, Gardens' Bulletin, Singapore 14: 190 (Sinclair 1955). - Lectotype (designated by Xue et al. 2012: 1032): Peninsular Malaysia, Perak, King's Collector [H.H. Kunstler] 6654 (hololecto-: L (L0037926); isolecto-: CAL, DD). 


\section{Description and distribution}

Sinclair (1955: 190-191).

Monoon magnoliiflorum (Maingay ex Hook.f. \& Thomson) B.Xue \& R.M.K.Saunders

Taxon 61: 1032 (Xue et al. 2012). - Polyalthia magnoliiflora Maingay ex Hook.f. \& Thomson, The Flora of British India 1: 64 (Hooker \& Thomson 1872). - Griffithia magnoliipetala King, Annals of the Royal Botanic Garden (Calcutta) 4: 9, t. 218 (King 1893), as 'magnoliaepetala', nom. superfl. Griffithianthus magnoliiflorus (Maingay ex Hook.f. \& Thomson) Merr., Philippine Journal of Science. Section C, Botany 10: 231 (Merrill 1915). - Enicosanthum magnoliiflorum (Maingay ex Hook.f. \& Thomson) Airy Shaw, Bulletin of Miscellaneous Information, Kew 1939: 277 (Airy-Shaw 1939). Lectotype (designated by Xue et al. 2012: 1032): Malacca, 6 Feb. 1868, A.C. Maingay 3259 [Kew distrib. no. 93] (K (K000691423)).

\section{Description and distribution}

Sinclair (1955: 187-188).

\section{Monoon malayanum I.M.Turner \& Utteridge}

Webbia 70: 99 (Turner \& Utteridge 2015). - Type: Peninsular Malaysia, Trengganu, Ulu Trengganu, Ulu Telemong Forest Reserve, Batu Kota, 16 Sep. 1969. H.S. Loh FRI 13446 (holo-: KEP; iso-: K [×2]).

\section{Description and distribution}

Turner \& Utteridge (2015: 99).

\section{Monoon membranifolium (J.Sinclair) B.Xue \& R.M.K.Saunders}

Taxon 61: 1032 (Xue et al. 2012). - Enicosanthum membranifolium J.Sinclair, Gardens' Bulletin, Singapore 14: 191 (Sinclair 1955). - Lectotype (designated by Xue et al. 2012: 1032): Peninsular Malaysia, Kelantan, Gua Panjang at Gua Ninik, 21 Oct. 1927, M.R. Henderson SFN 19522 (hololecto-: SING (SING0048664); isolecto-: K (K000691413), SING (SING0048665)).

\section{Description and distribution}

Sinclair (1955: 191).

Monoon pachyphyllum (King) B.Xue \& R.M.K.Saunders

Taxon 61: 1033 (Xue et al. 2012). - Polyalthia pachyphylla King, Journal of the Asiatic Society of Bengal. Part II (Natural History \& c.) 61: 66 (King 1892). - Lectotype (designated by Xue et al. 2012: 1033): Peninsular Malaysia, Perak, King's Collector [H.H. Kunstler] 6655 (hololecto-: K (barcode no. K000691559); isolecto-: BM (BM000754027), CAL, DD, SING).

\section{Description and distribution}

Sinclair (1955: 308).

\section{Monoon praestigiosum (J.Sinclair) B.Xue \& R.M.K.Saunders}

Taxon 61: 1033 (Xue et al. 2012). - Enicosanthum praestigiosum J.Sinclair, Gardens'Bulletin, Singapore 14: 192 (Sinclair 1955). - Lectotype (designated by Xue et al. 2012: 1032): Peninsular Malaysia, 
TURNER I.M. \& UTTERIDGE T.M.A., Whither Polyalthia (Annonaceae) in Peninsular Malaysia?

Johore, Sungei Kayu, 10 Oct. 1936, Kiah SFN 32017 (hololecto-: SING (SING00048666); isolecto-: A (A00039311), BO, BKF, K [×2] (K000691412, K000898010), KEP (KEP 8626), L, LAE, PNH).

\section{Description and distribution}

Sinclair (1955: 192-194).

Monoon sclerophyllum (Hook.f. \& Thomson) B.Xue \& R.M.K.Saunders

Taxon 61: 1033 (Xue et al. 2012). - Polyalthia sclerophylla Hook.f. \& Thomson, The Flora of British India 1: 65 (Hooker \& Thomson 1872). - Lectotype (designated here): Peninsular Malaysia, Malacca, 9 May 1867, A.C. Maingay 1340 [Kew distrib. no. 101] (hololecto-: K [×2] (K000691575, K000691576)). Polyalthia purpurea Ridl., Journal of the Straits Branch of the Royal Asiatic Society 82: 168 (Ridley 1920). - Lectotype (designated by Turner 2012: 242): Singapore, cultivated in Singapore Botanic Gardens, Jun. 1904, H.N. Ridley 12014 (hololecto-: K (K000691573); isolecto-: SING).

\section{Description and distribution}

Sinclair (1955: 311-312).

\section{Monoon xanthopetalum (Merr.) B.Xue \& R.M.K.Saunders}

Taxon 61: 1034 (Xue et al. 2012). - Polyalthia xanthopetala Merr., Journal of the Straits Branch of the Royal Asiatic Society 85: 185 (Merrill 1922). - Lectotype (designated by Turner 2011: 80): Borneo, Sabah, Batu Lima near Sandakan, Sep.-Dec. 1920, M. Ramos 1705 (hololecto-: K (K0006916180); isolecto-: A (A00242651), BM (BM000754105), BO, L (L0189515), US (US00098685)).

\section{Remarks}

In studying material of Monoon from Peninsular Malaysia a group of specimens was found that did not match any of the species recorded from this area. The specimens were notable for the persistent goldenbrown tomentum on the twigs, the often dark brown shade of the dry leaves, petals linear-lanceolate $c$. $4 \mathrm{~cm}$ long and c. $5 \mathrm{~mm}$ wide, monocarps ellipsoidal, covered with a short dense pale brown tomentum with the stipe attached slightly eccentrically giving a similar appearance to those of some Uvaria species previously included in the genus Ellipeia. One of us (IMT) having worked on the Borneo species (Turner 2014), the specimens were reminiscent of Monoon xanthopetalum Merr. Comparison of the specimens from Peninsular Malaysia with collections from Borneo (cf. Turner 2014: Fig. 26) produced a close match. We have no doubt that Monoon xanthopetalum does occur in Peninsular Malaysia, representing a major range extension for the species. It is interesting to note that the Peninsular Malaysia collections of M. xanthopetalum are all from the East Coast which fits with the presence of the species in Borneo.

\section{Specimens examined}

PENINSUlAR MALAYSIA: Johore, Mersing, Hutan Simpan Lenggor, 2 Nov. 2010, L.E. Teo \& Din KL 5758, (KEP). PaHANG, Rompin, Gunong Lesong Forest Rerve, 2 May 1956, Forester Lindong KEP 83466 (K); Lesong Forest Reserve, 27 Jun. 1972, Y.C. Chan FRI 19827 (K, KEP). Trengganu, 26 Oct. 1994, C. Wiart \& L.E. Teo KL 4434 (KEP); Kemaman, Kajang, Ulu Bendong, 6 Nov. 1935, E.J.H. Corner SFN 30291 (K).

\section{Polyalthia Blume}

Flora Javae (Anonaceae): 68 (Blume 1830). - Lectotype (designated by Hutchinson 1923: 259): Polyalthia subcordata (Blume) Blume. 
Sphaerothalamus Hook.f., Transactions of the Linnean Society of London 23: 156 (Hooker 1860). Type: Sphaerothalamus insignis Hook.f.

\section{Key to the species of Polyalthia}

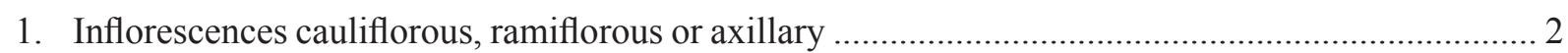

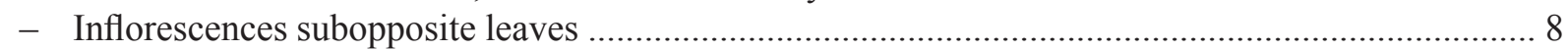

2. Flower pedicel $2-5(-7) \mathrm{mm}$ long; petals narrowly linear (generally more than 10 times long as broad). Monocarps subsessile (stipes to $5 \mathrm{~mm}$ long)

- Flower pedicel (6-)10-30 mm long; petals not narrowly linear (rarely to 10 times as long as broad). Monocarps stipitate (stipes $10 \mathrm{~mm}$ long or more).

3. Subshrub to $30 \mathrm{~cm}$ tall. Petals not exceeding $3 \mathrm{~cm}$ long

P. pumila

- Tree to $6 \mathrm{~m}$ or more tall. Petals to $5 \mathrm{~cm}$ or more long ... 4

4. Petals $5 \mathrm{~mm}$ wide or more. Monocarps densely brown woolly hairy P. cinnamomea

- Petals 2-5 $\mathrm{mm}$ wide. Monocarps pubescent, becoming glabrous with maturity ...... 5

5. Inflorescences mostly on trunk and branches. Twigs persistently brown hairy. Sepals $15-17 \mathrm{~mm}$ long. Monocarps $2 \mathrm{~cm}$ wide

- Inflorescences mostly among leaves. Twigs becoming glabous with age. Sepals $7 \mathrm{~mm}$ long. Monocarps less than $1 \mathrm{~cm}$ wide

P. angustissima

6. Inflorescences mostly cauliflorous, or if among leaves then pedicel generally $20 \mathrm{~mm}$ long or more ..... P. cauliflora

- Inforescences not cauliflorous, flower pedicel to $12 \mathrm{~mm}$ long

7. Leaves drying grey. Flower pedicel more than $1 \mathrm{~mm}$ wide, widening distally; petals $2-5 \mathrm{~cm}$ long. Monocarps more or less cylindrical, 6-7 mm diameter; stipe $c .1 \mathrm{~mm}$ diameter

P. lateritia

- Leaves drying brown or grey-brown. Flower pedicel c. $0.5 \mathrm{~mm}$ wide; petals less than $1 \mathrm{~cm}$ long. Monocarps more or less globose, $c .15 \mathrm{~mm}$ diameter; stipe $c .2 \mathrm{~mm}$ diameter.

P. obliqua

8. Leaves with many lateral nerves (at least 18 pairs); lamina bullate ............................................... 9

- Leaves with up to 17 pairs of lateral nerves; lamina not bullate .................................................... 10

9. Twigs villose. Base of leaf auriculate or cordate, often extending below twig

P. bullata

- Twigs glabrous or hairy, not villose. Base of leaf not auriculate or cordate, never extending below twig

P. pakdin I.M.Turner \& Utteridge sp. nov.

10. Leaves narrowly elliptic or lanceolate to $2.5 \mathrm{~cm}$ wide, apex long acuminate

P. dumosa

- Leaves generally more than $2.5 \mathrm{~cm}$ wide, apex not long acuminate 11

11. Leaf apex obtuse. Branchlets with thick corky bark. Flower pedicel slender (5-25 mm long, $<1 \mathrm{~mm}$ thick) .......

- Leaf apex generally acute to acuminate. Branchlets not corky-barked. Flower pedicels generally shorter and/or thicker

12. Flowers subsessile (pedicel to $2 \mathrm{~mm}$ long)

- Flowers distinctly pedicellate (pedicel $5 \mathrm{~mm}$ long or more)

13. Leaves glabrous; elliptic-lanceolate; apex blunt. Petals coriaceous, fleshy

P. parviflora 
- Leaves pubescent beneath; lanceolate; acuminate. Petals thin P. hirtifolia

14. Twigs, leaf undersides, flowers and fruits furry P. chrysotricha

- Twigs, leaf undersides, flowers and fruits glabrous or hairy but not all furry 15

15. Leaves generally with 10 or fewer pairs of lateral nerves. Flower pedicel to $5 \mathrm{~mm}$ long. Monocarps to 6 , stipe shorter than seed-bearing portion

P. brunneifolia

- Leaves with 12 or more pairs of lateral nerves. Flower pedicel $5 \mathrm{~mm}$ or more long. Monocarps 10 or more, stipe generally longer than seed-bearing portion

16. Petals relatively thin; sparsely hairy outside. Stipe of monocarps $c .0 .5 \mathrm{~mm}$ thick P. socia

- Petals relatively thick and leathery; densely hairy outside. Stipe of monocarps $c .1 \mathrm{~mm}$ thick 17

17. Pedicel 3-6 cm long P. oblonga

- Pedicel to $1 \mathrm{~cm}$ long P. motleyana

\section{Polyalthia angustissima Ridl.}

Journal of the Straits Branch of the Royal Asiatic Society 54: 11 (Ridley 1910). - Lectotype (designated by Bunchalee \& Chantaranothai 2006: 3): Singapore: Bukit Timah, Feb. 1896, H.N. Ridley 8050 (hololecto-: SING (SING0048677); isolecto-: K).

\section{Description and distribution}

Sinclair (1955: 286).

\section{Polyalthia brunneifolia J.Sinclair}

Gardens'Bulletin, Singapore 14: 301 (Sinclair 1955). - Lectotype (designated here): Peninsular Malaysia, Selangor, Bukit Enggang, Kajang, 8 Apr. 1930, C.F. Symington 24179 (hololecto-: K (K000691554); isolecto-: SING (SING0048680).

\section{Description and distribution}

Sinclair (1955: 301-302).

\section{Polyalthia bullata King}

Journal of the Asiatic Society of Bengal. Part II (Natural History \&c.) 61: 64 (King 1892). - Lectotype (designated by Turner 2009: 92): Peninsular Malaysia, Perak, Gopeng, Kinta, Aug. 1883, King's Collector [H.H. Kunstler] 4804 (hololecto-: CAL; isolecto-: K (K000691434)).

\section{Description and distribution}

Sinclair (1955: 300-301), Turner (2014: 145).

\section{Polyalthia cauliflora Hook.f. \& Thomson}

Flora Indica: 138 (Hooker \& Thomson 1855). - Type: Singapore, Oct. 1822, N. Wallich s.n. [EIC 6476] (holo-: K-W (K001123992)).

Guatteria teysmannii Miq., Flora van Nederlandsch Indië. Supplementum primum. Prodromus florae sumatranae: 378 (Miquel 1861). - Monoon teysmannii (Miq.) Miq., Annales Musei Botanici LugdunuBatavi 2: 19 (Miquel 1865). - Polyalthia teysmannii (Miq.) King, Journal of the Asiatic Society of Bengal. Part II (Natural History \&c.) 61: 67 (King 1892). - Lectotype (designated by Turner 2011: 
69): Indonesia, Sumatra, Palembang, Batu Radja, s. dat., J.E. Teijsmann s.n. [Herb. Bogor. 3901] (U (U 0000373)).

Unona desmantha Hook.f. \& Thomson, The flora of British India 1: 61 (Hooker \& Thomson 1872).Desmos desmanthus (Hook.f. \& Thomson) Saff., Bulletin of the Torrey Botanical Club 39: 508 (Safford 1912). - Polyalthia cauliflora var. desmantha (Hook.f. \& Thomson) J.Sinclair, Gardens' Bulletin, Singapore 14: 295 (Sinclair 1955). - Lectotype (designated by Turner 2010b: 268): Malaysia, Peninsular Malaysia, Malacca, 1865-1866, A.C. Maingay 1045 [Kew distribution no. 48] (K (K000691431)).

Polyalthia beccarii King, Journal of the Asiatic Society of Bengal. Part II (Natural History \&c.) 61: 65 (King 1892). - Polyalthia cauliflora Hook.f. \& Thomson var. beccarii (King) J.Sinclair, Gardens' Bulletin, Singapore 14: 294 (Sinclair 1955). - Lectotype (designated by Turner 2009: 93): Malaysia, Peninsular Malaysia, Perak, Gopeng, Kinta, Jul. 1883, King's Collector [H.H. Kunstler] 4522 (hololecto-: K (K00069131); isolecto-: CAL, DD, U).

Unona pycnantha Hook.f. \& Thomson, The Flora of British India 1: 60 (Hooker \& Thomson 1872). - Polyalthia pycnantha (Hook.f. \& Thomson) King, Journal of the Asiatic Society of Bengal. Part II (Natural History \&c.) 61: 67 (King 1892). - Lectotype (designated by Turner 2010b: 268): Malaysia, Peninsular Malaysia: Malacca, 9 Jan. 1865 or 1866, A.C. Maingay 1491 [Kew distribution no. 48] (K (K000691459)).

Unona wrayi Hemsl., Hooker's Icones Plantarum 16: t. 1553 (Hemsley 1887). - Polyalthia wrayi (Hemsl.) Ridl., The Flora of the Malay Peninsula 1: 49 (Ridley 1922). - Polyalthia cauliflora Hook.f. \& Thomson var. wrayi (Hemsl.) J.Sinclair, Gardens'Bulletin, Singapore 14: 296 (Sinclair 1955). - Type: Peninsular Malaysia, Perak, Ulu Kenring, Jan. 1884, L. Wray 560 (holo-: K (K000691466)).

\section{Description and distribution}

Sinclair (1955: 292-297), Turner (2014: 145-146).

\section{Polyalthia chrysotricha Ridl.}

The Flora of the Malay Peninsula 1: 57 (Ridley 1922). - Type: Peninsular Malaysia, Selangor, Ulu Langat, Menuang Gasing, Feb. 1912, C.B. Kloss s.n. (holo-: K (K000691577)).

\section{Description and distribution}

Sinclair (1955: 302-303), Turner (2014: 147).

Polyalthia cinnamomea Hook.f. \& Thomson

Flora Indica: 138 (Hooker \& Thomson 1855). - Lectotype (designated by Airy-Shaw 1939: 282): Singapore, Anon. [?N. Wallich] s.n. [EIC 6444] (hololecto-: K-W (K001123933)).

Unona cauliflora Hook.f. \& Thomson, Flora Indica: 137 (Hooker \& Thomson 1855), non U. cauliflora Blanco (Blanco 1845). - Type: Singapore, T. Lobb s.n. (holo-: K (K000691457) [excl. material in packet from EIC $=$ K000691458]).

Polyalthia velutinosa Ridl., Journal of the Straits Branch of the Royal Asiatic Society 59: 64 (Ridley 1911). - Lectotype (designated here): Peninsular Malaysia, Lankawi, Gunong Raya at 1500 feet, Feb. 1911, (Aniff) Haniff 15552 (hololecto-: SING (SING 0058808); isolecto-: BM, K (K000691439), BM).

\section{Description and distribution}

Sinclair (1955: 286-288), Kochummen (1972: 87-88), Turner (2014: 148). 


\section{Polyalthia dumosa King}

Journal of the Asiatic Society of Bengal. Part II (Natural History \&c.) 61: 52 (King 1892). - Lectotype (designated by Bunchalee \& Chantaranothai 2006: 3): Peninsular Malaysia, Perak, Maxwell's Hill, $L$. Wray 2628 (hololecto-: SING; isolecto-: CAL).

\section{Description and distribution}

Sinclair (1955: 297).

\section{Polyalthia hirtifolia J.Sinclair}

Gardens' Bulletin, Singapore 14: 300 (Sinclair 1955). - Polyalthia hirta Ridl., Journal of the Straits Branch of the Royal Asiatic Society 82: 168 (Ridley 1920), non P. hirta (Miq.) Diels (Diels 1912). - Lectotype (designated by Turner 2012: 241): Peninsular Malaysia, Penang, Pulau Butong Reserve, Mar. 1892, C. Curtis 2745 (hololecto-: K (K000691452); isolecto-: BM, K (barcode no. K000691453), SING).

\section{Description and distribution}

Sinclair (1955: 300).

\section{Polyalthia lateritia J.Sinclair}

Gardens'Bulletin, Singapore 14: 290 (Sinclair 1955). - Type: Peninsular Malaysia, Perak, Waterloo New Road, May 1890, C. Curtis 2704 (holo-: SING (SING0048683); iso-: K (K000691549), SING (SING0051296)).

\section{Description and distribution}

Sinclair (1955: 290-291),

Polyalthia motleyana (Hook.f.) Airy Shaw

Bulletin of Miscellaneous Information, Kew 1939: 280 (Airy-Shaw 1939). - Oxymitra motleyana Hook.f., Transactions of the Linnean Society of London 23: 155 (Hooker 1860), as 'molleyana'. - Lectotype (designated by Turner 2011: 76): Borneo, Labuan, E.S. Barber 228 (hololecto-: K (K000691612); isolecto-: K (K000691613)).

Polyalthia sarawakensis Diels, Notizblatt des Botanischen Gartens und Museums zu Berlin-Dahlem 11: 80 (Diels 1931). - Type: Borneo, Sarawak, Mt Mattan, Apr. 1866, O. Beccari P.B. 1623 (holo-: FI-B (Erbario collesioni Beccari no. 415)).

Polyalthia motleyana var. glabrescens Airy Shaw, Bulletin of Miscellaneous Information, Kew 1939: 282 (Airy-Shaw 1939). - Type: Borneo, Sarawak, O. Beccari P.B. 1813 (holo-: K (K000691611); iso-: FI-B).

\section{Description and distribution}

Sinclair (1955: 303-305), Turner (2014: 157-159).

\section{Polyalthia obliqua Hook.f. \& Thomson}

Flora Indica: 138 (Hooker \& Thomson 1855). - Lectotype (designated by Turner 2011: 77): Peninsular Malaysia, Malacca, s. dat., W. Griffith s.n. (K (K000691435)). 
Polyalthia similis Merr., Philippine Journal of Science. Section C, Botany 8: 371 (Merrill 1913). Lectotype (designated by Turner 2011: 77): Philippines, Leyte, Dagami, Aug. 1912, M. Ramos Bur. Sci. 15185 (hololecto-: K (K000691620); isolecto-: BM, US (US00098679)).

Polyalthia lopadantha Diels, Notizblatt des Botanischen Gartens und Museums zu Berlin-Dahlem 11: 81 (Diels 1931). - Type: Borneo, Sarawak, O. Beccari P.B. 1819 (holo-: FI-B (Erb. coll. Becc. no. 419); iso-: $\mathrm{A}[\times 2], \mathrm{B}[\times 2](\mathrm{B} 10$ 0186733, B 100186734 (fragment)), K (K000691615), M).

\section{Description and distribution}

Sinclair (1955: 290), Turner (2014: 159-160).

\section{Polyalthia oblonga King}

Journal of the Asiatic Society of Bengal. Part II (Natural History \&c.) 61: 65 (King 1892). - Polyalthia motleyena var. oblonga (King) J.Sinclair, Gardens' Bulletin, Singapore 14: 304 (Sinclair 1955). - Lectotype (designated by Turner 2011: 77): Peninsular Malaysia, Perak, Larut, May 1885, King's Collector [H. H. Kunstler] 7671 (hololecto-: K; isolecto-: CAL, DD, SING).

\section{Description and distribution}

Sinclair (1955: 304), Turner (2014: 160).

Polyalthia pakdin I.M.Turner \& Utteridge sp. nov.

Figs $1-2$

urn:lsid:ipni.org:names:77153637-1

\section{Diagnosis}

With its large, multi-nerved leaves the new species has generally been confused with Polyaltha bullata King, but it lacks the villose tomentum on the twigs and the strongly auriculate to cordate leaf bases. In comparison with $P$. bullata, the flowers are borne on shorter pedicels $(<1$ versus $2 \mathrm{~cm}$ ) with shorter sepals (3-4 versus $7 \mathrm{~mm}$ ) and petals (to $1 \mathrm{~cm}$ versus up to $4 \mathrm{~cm}$ ); and with monocarps on shorter stipes (to $6 \mathrm{~mm}$ versus up to $10 \mathrm{~mm}$ ).

\section{Etymology}

We are pleased to commemorate the late Prof. Kamarudin Mat Salleh (1959-2009). A leading Malaysian student of the Annonaceae, he was widely known as 'Pak Din' (Uncle Din in Malay, Din being the common abbreviation of names with that ending). The epithet represents a compounding of this soubriquet. Kamarudin was native to the state of Kelantan, which is part of the range of the new species.

\section{Type}

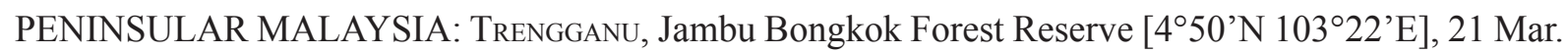
1972, L.E. Teo \& G. Pachiappan KL 3033 [T \& P 433] (holo-: K).

\section{Additional specimens examined}

PENINSULAR MALAYSIA: KelANTAN, Chabang Tongkat Forest Reserve [5 ${ }^{\circ} 49^{\prime} \mathrm{N} 102^{\circ} 17^{\prime} \mathrm{E}$ ], compt 77, alt. 250' 28 Apr. 1967, K.M. Kochummen FRI 2317 (KEP). TRengGanu, Dunggun, Jambu Bongkok [450'N 10321'E], 12 Mar. 1961, J. Carrick s.n. (K ×2); ibid., Sekayu, Bukit Lanjut Forest Reserve [458'N 102 58'E], 18 Sept. 1969, H.S. Loh FRI 13467 (KEP); ibid., Dungun, near 36th mile, Jerangau Road [456’36”N 10310’42”E], 22 Sept. 1955, J. Sinclair \& Kiah bin Salleh SFN 40941 (K, KEP); ibid., Sekayu Forest Reserve [458’N 10257'E], compt 43, 22 Sept. 1969, T. Suppiah FRI 11853 (KEP); 


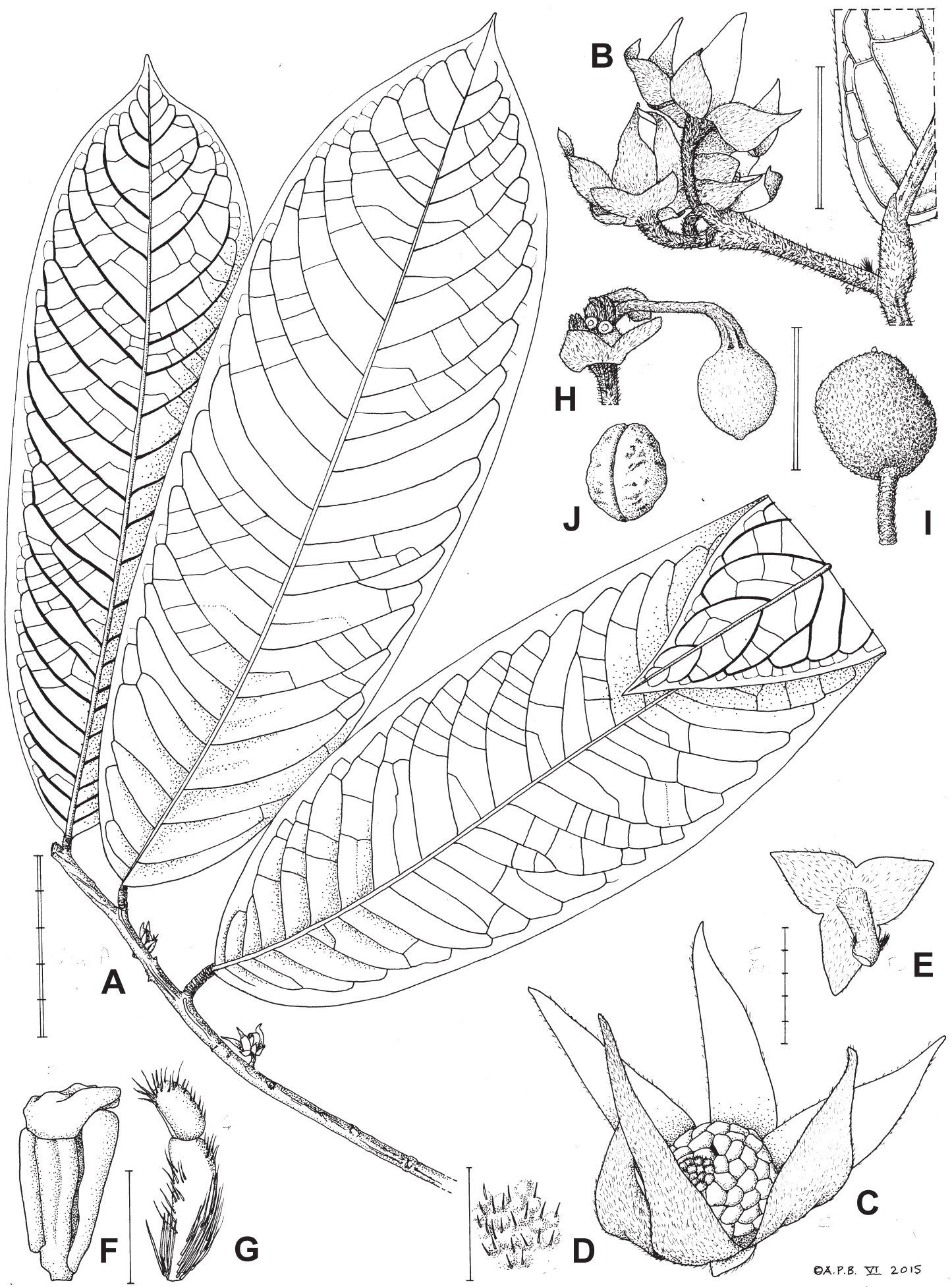

Fig. 1. Polyalthia pakdin I.M.Turner \& Utteridge sp. nov. A. Leafy twig with flowers. B. Inflorescence and base of leaf (abaxial view). C. Flower. D. Magnified view of abaxial surface of outer petal. E. Calyx from below. F. Stamen. G. Carpel. H. Infructescense with immature monocarp. I. Mature monocarp. J. Seed. Scale bars: single bar $=1 \mathrm{~mm}$, graduated single bar $=5 \mathrm{~mm}$; double bar $=1 \mathrm{~cm}$, graduated double bar $=5$ cm. Drawn from Carricks.n. (A, C-E), KL 3033 (B, F, G), SFN 40941 (H), FRI 11853 (I), FRI $13467(\mathrm{~J})$. 


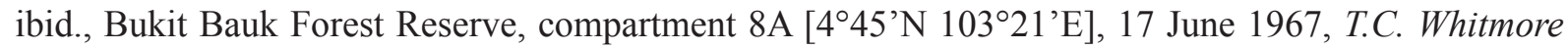
FRI 3903 (KEP).

\section{Description}

Shrub 1-4 m tall. Young twigs drying red-brown, rather smooth and shiny, with some irregular longitudinal ridges with rounded tops, varying from glabrous to densely pale short hairy, twigs becoming black to brown with age. Leaves chartaceous to subcoriaceous (tend to look more leathery than they are to the touch), often bullate, drying grey or grey-brown, more rarely dark brown, above, brown or greybrown below with brown venation, midrib and laterals sunken above in dry leaves, prominent beneath, more or less glabrous though sometimes with pale hairs along midrib below near base, lamina oblongoblanceolate to narrowly oblong-elliptic, $17-36 \times 3.5-9 \mathrm{~cm}$, apex acuminate, base obtuse, generally slightly asymmetric, lateral nerves 19-24 pairs, arching and looping within margin to form a scalloped intramarginal nerve, reticulations distinct. Petiole drying dark brown to black, 6-10 mm long, 2-4 mm in diameter, sometimes with short erect pale hairs. Inflorescences subopposite leaves, single- or fewflowered. Flowering pedicel 2-8 $\mathrm{mm}$ long, $1 \mathrm{~mm}$ diameter widening distally, drying brown with minute pale hairs [some 4-merous flowers present on $K L$ 3033], sepals ovate $c$. 3-4 4 2-3 mm, outside brown, minutely bumpy with short pale hairs, inside glabrous, petals thin-textured, ovate to ovate-lanceolate 6-10 $\times 2.5-3 \mathrm{~mm}$, drying dark brown to blackish, outside minutely bumpy with adpressed pale hairs, inside glabrous, stamens many c. $1.5 \mathrm{~mm}$ long, carpels many. Fruiting pedicel to $2 \mathrm{~mm}$ thick, sepals often persisting, monocarps to 9 or more, irregularly ellipsoidal, $8-10 \times 7-9 \mathrm{~mm}$, drying red-brown, surface minutely pimply with pale hairs, apiculate, stipe to $6 \mathrm{~mm}$ long, $1 \mathrm{~mm}$ diameter, pimply, pale hairy. Seeds generally two, lenticular, $c .7 \times 6 \times 4 \mathrm{~mm}$, drying golden brown with a longitudinal groove running parallel to the flat face.

\section{Field notes}

Leaves bullate (SFN 40941), dark glossy green above, paler green beneath (SFN 40941), shiny dark green above, slightly yellowish green below (KL 3033); flowers yellowish $(K L 3033)$, petals reddish orange (SFN 40941), petals and sepals orange (FRI 2317), stamens paler reddish orange (SFN 40941), no scent with flowers (SFN 40941); fruits green (FRI 3903), fruits green turning red (FRI 11853), fruits red (FRI 13467, SFN 40941); seeds brown (SFN 40941).

\section{Distribution and habitat}

Recorded from the eastern side of Peninsular Malaysia from Kelantan and, with most records, Trengganu (Fig. 2). It occurs in lowland tropical rain forest.

\section{Conservation status}

Polyalthia pakdin sp. nov. is restricted to the lowland forests of eastern Peninsular Malaysia and currently known from seven collections from five localities (two are from the Sekayu area and two from Jambu Bongkok). The Extent of Occurrence (EOO) is $1,856 \mathrm{~km}^{2}$ falling within the Endangered (EN) threshold of less than $5,000 \mathrm{~km}^{2}$, and the Area of Occupancy (AOO; based on a user-defined cell width of $2 \mathrm{~km}$ ) is $28 \mathrm{~km}^{2}$ falling within the Endangered (EN) threshold. Whilst none of the collections is contemporary (the most recent is 1972), nearly all are from forests reserves, and, in addition, one collection is from 'roadside forest' (J. Sinclair \& Kiah bin Salleh SFN 40941). Whilst the EOO is within the EN threshold, the presence of the species within protected areas would not place the species within this category because recent satellite imagery shows the protected areas still with relatively large areas of intact forest (using the most recent Google Earth imagery). The Sekayu collections, for example, are on the edge of the very poorly known and under-collected Taman Negara National Park and more field collections are needed in such areas to better understand the distribution of this species. Without any other information, we must assume that the species is still present in these protected areas and that the protected areas still 
harbour the appropriate habitat for P. pakdin sp. nov. It is difficult to demonstrate trends in the reduction of habitat etc. without modern collections, and for these reasons we assign a preliminary conservation assessment of Near Threatened (NT).

\section{Affinities}

Polyalthia bullata, the species with which P. pakdin sp. nov. was formerly confused, was included in the informal group of Polyalthia species termed the Polyalthia insignis group by Johnson \& Murray (1999). They characterised the group as follows: shrubs or small trees; leaves oblique, subcordate or auriculate at base, often drying grey above, brown below; inflorescences internodal to leaf opposed or sometimes cauliflorous; petals orange or red; apex of ovary woody and persistant as a short beak on the monocarp; stigmas capitate and cohering to drop as a cap at the end of anthesis, several ovules per carpel, laterally attached in vertical row and seed not pitted but with encircling groove. While Polyalthia pakdin sp. nov. does exhibit some of these characters, it does not have auriculate or cordate leaf bases and the stigmas do not seem to cohere. In fact, P. pakdin sp. nov. also comes close to the Bornean Polyalthia polyphlebia Diels which was not included in the $P$. insignis group by Johnson \& Murray. The leaf form of $P$. pakdin sp. nov. and $P$. polyphlebia are similar but $P$. polyphlebia generally has greyish corky twigs, longer pedicels ( $8-15$ versus $2-8 \mathrm{~mm}$ ) with the flowers larger (outer petals $13-14 \mathrm{~mm}$ versus $6-10 \mathrm{~mm}$ ) and the petals fleshy rather than thin in texture; with the monocarps on longer stipes (to $25 \mathrm{~mm}$ versus $6 \mathrm{~mm}$ ).

\section{Polyalthia parviflora Ridl.}

Journal of the Straits Branch of the Royal Asiatic Society 61: 49 (Ridley 1912). - Lectotype (designated by Turner 2012: 241): Peninsular Malaysia, Pulau Langkawi, Kuala Malacca, Sep. 1890, C. Curtis 2533 (hololecto-: SING (SING 0059296); isolecto-: CAL).

Polyalthia rufa Craib, Bulletin of Miscellaneous Information, Kew 1924: 82 (Craib 1924). - Type: Thailand, Nakawn Sawawn, Mê Wong, 25 May 1922, A.F.G. Kerr 6022 (holo-: K[×2] (K00595497, K00595496); iso-: ABD, BM (000553972(BM))).

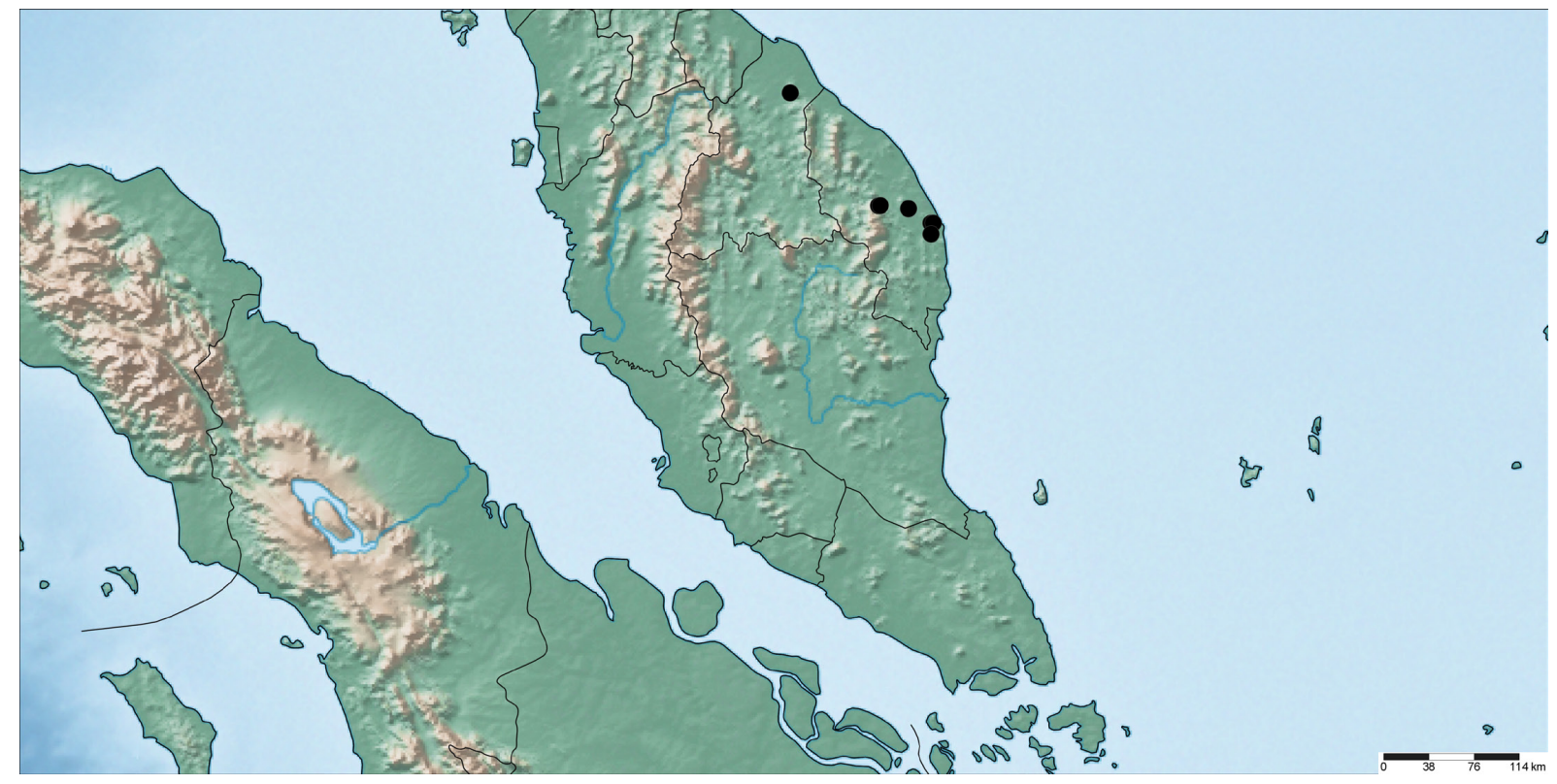

Fig. 2. Map showing the collecting localities of Polyalthia pakdin I.M.Turner \& Utteridge sp. nov. 


\section{Description and distribution}

Sinclair (1955: 299).

\section{Polyalthia pumila Ridl.}

Journal of the Straits Branch of the Royal Asiatic Society 54: 12 (Ridley 1910). - Lectotype (designated by Turner 2012: 241): Peninsular Malaysia, Perak, Dindings, Sera Woods, Mar. 1896, H.N. Ridley 7996 (hololecto-: SING (SING 0059298); isolecto-: K (K000691442)).

\section{Description and distribution}

Sinclair (1955: 289).

\section{Polyalthia socia Craib}

Bulletin of Miscellaneous Information, Kew 1925: 10 (Craib 1925). - Type: Thailand, Pattani, Bukit, 400 m, evergreen forest, 7 Jul. 1923, A.F.G. Kerr 7101 (holo-: K (K000595494); iso-: ABD[×2], BK (257670), BM (000553974(BM)), TCD (TCD0009808)).

\section{Description and distribution}

Sinclair (1955: 291-292).

Polyalthia stenopetala (Hook.f. \& Thomson) Finet \& Gagnep.

Mémoires de la Société Botanique de France 4: 96 (Finet \& Gagnepain 1906). - Unona stenopetala Hook.f. \& Thomson, Flora Indica: 136 (Hooker \& Thomson 1855). - Desmos stenopetalus (Hook.f. \& Thomson) Saff., Bulletin of the Torrey Botanical Club 39: 507 (Safford 1912). - Lectotype (designated by Sinclair 1955: 285): Java, T. Lobb 414 (hololecto-: K (K000691463); isolecto-: CGE).

Unona crinita Hook.f. \& Thomson, The Flora of British India 1: 61 (Hooker \& Thomson 1872). Desmos crinitus (Hook.f. \& Thomson) Saff., Bulletin of the Torrey Botanical Club 39: 507 (Safford 1912). - Polyalthia crinita (Hook.f. \& Thomson) Ridl., Journal of the Straits Branch of the Royal Asiatic Society 75: 6 (Ridley 1917). - Type: Peninsular Malaysia, Malacca, 18 Jan. 1865 or 1866, A. C. Maingay 1513 [Kew Distribution no. 41] (holotype: K[×2] (K000691461, K000691462)).

\section{Description and distribution}

Sinclair (1955: 285), Turner (2014: 162).

Polyalthia suberosa (Roxb.) Thwaites

Enumeratio Plantarum Zeylaniae 5: 398 (Thwaites 1864). - Uvaria suberosa Roxb., Plants of the Coast of Coromandel 1: 31, t. 34 (Roxburgh 1795). - Guatteria suberosa (Roxb.) Dunal, Monographie de la famille des Anonacées: 128 (Dunal 1817). - Lectotype (designated by Huber 1985: 41): Roxburgh's plate t. 34.

\section{Description and distribution}

Sinclair (1955: 298).

\section{Discussion}

The changes in the species recognised for Peninsular Malaysia between Sinclair's revision and the current compilation are summarised in Table 1. Three species have been added to the list in the 
Table 1. Comparison of accepted names of species in Polyalthia sensu lato native to Peninsular Malaysia between Sinclair (1955) and the current paper.

\begin{tabular}{|c|c|}
\hline Sinclair 1955 & Current Paper \\
\hline Enicosanthum congregatum & Monoon congregatum \\
\hline Enicosanthum cupulare & Monoon cupulare \\
\hline Enicosanthum fuscum & Monoon fuscum \\
\hline Enicosanthum macranthum & Monoon macranthum \\
\hline Enicosanthum magnoliiflorum & Monoon magnoliiflorum \\
\hline Enicosanthum membranifolium & Monoon membranifolium \\
\hline Enicosanthum praestigiosum & Monoon praestigiosum \\
\hline Polyalthia jenkinsii & Huberantha jenkinsii \\
\hline Polyalthia rumphii & Huberantha rumphii \\
\hline Polyalthia glauca & Maasia glauca \\
\hline Polyalthia hypoleuca & Maasia hypoleuca \\
\hline Polyalthia sumatrana & Maasia sumatrana \\
\hline Polyalthia asteriella & Monoon asteriellum \\
\hline Polyalthia clavigera & Monoon anomalum \\
\hline Polyalthia glabra & Monoon glabrum \\
\hline Polyalthia hookeriana & Monoon hookerianum \\
\hline Polyalthia hypogaea & Monoon hypogaeum \\
\hline Polyalthia lateriflora & Monoon lateriflorum \\
\hline Polyalthia macropoda & Monoon borneense \\
\hline Polyalthia pachyphylla & Monoon pachyphyllum \\
\hline Polyalthia sclerophylla & Monoon sclerophyllum \\
\hline - & Monoon malayanum \\
\hline - & Monoon xanthopetalum \\
\hline Polyalthia angustissima & Polyalthia angustissima \\
\hline Polyalthia bullata & Polyalthia bullata \\
\hline Polyalthia brunneifolia & Polyalthia brunneifolia \\
\hline Polyalthia cauliflora & Polyalthia cauliflora \\
\hline Polyalthia chrysotricha & Polyalthia chrysotricha \\
\hline Polyalthia cinnamomea & Polyalthia cinnamomea \\
\hline Polyalthia dumosa & Polyalthia dumosa \\
\hline Polyalthia hirtifolia & Polyalthia hirtifolia \\
\hline Polyalthia lateritia & Polyalthia lateritia \\
\hline Polyalthia motleyana & Polyalthia motleyana \\
\hline Polyalthia motleyana var. oblonga & Polyalthia oblonga \\
\hline Polyalthia obliqua & Polyalthia obliqua \\
\hline Polyalthia parviflora & Polyalthia parviflora \\
\hline Polyalthia pumila & Polyalthia pumila \\
\hline Polyalthia socia & Polyalthia socia \\
\hline Polyalthia stenopetala & Polyalthia stenopetala \\
\hline Polyalthia suberosa & Polyalthia suberosa \\
\hline & Polyalthia pakdin sp. nov. \\
\hline
\end{tabular}


intervening 60 years which is perhaps fewer than might be expected and must reflect Sinclair's thorough study of the specimens available to him. There have been a large number of name changes, with fewer than half the species still having the same accepted name - all in Polyalthia s.s. The reason for this is primarily to create a more phylogenetically accurate taxonomy. It is clear that Polyalthia sensu lato was heterogeneous: uniting species with a roughly similar floral morphology. Users of taxonomy, such as foresters and ecologists, generally dislike name changes so there may not be much enthusiasm from them after these changes. The splitting up of Polyalthia will possibly have some practical advantages as large genera can be unwieldy and daunting to non-specialists. However, expanding the list of genera in a family that already has a large number to deal with may also discourage the users of taxonomy. Fortunately in this case, one of the three 'new' genera - Maasia - is easy to recognise because of the white or glaucous underside to the leaves giving a consistent gestallt to specimens that is soon learnt. Monoon and Polyalthia sensu stricto can be distinguished fairly readily, though some would doubtless have preferred the continued use of Enicosanthum rather than the resurrection of Monoon.

\section{Acknowledgements}

We thank the Curator of the FRIM herbarium for the loan of material and Andrew Brown for the excellent drawing of the new species. Constructive comments from two anonymous reviewers helped improve the paper.

\section{References}

Airy-Shaw H.K. 1939. Additions to the flora of Borneo and other Malay Islands: XII. The Annonaceae of the Oxford University Expedition to Sarawak, 1932. Bulletin of Miscellaneous Information Kew 1939: 275-290. http://dx.doi.org/10.2307/4111738

Bachman S., Moat J., Hill A., de la Torre J. \& Scott B. 2011. Supporting Red List threat assessments with GeoCAT: Geospatial Conservation Assessment Tool. ZooKeys 150: 117-126. http://dx.doi.org/10.3897/ zookeys.150.2109

Beccari O. 1871. Illustrazione di nuove o rare specie di piante Bornensi - Anonacee. Nuovo Giornale Botanico Italiano 3: 177-193.

Blanco F.M. 1845. Flora de Filipinas. Second edition. D. Miguel Sanchez, Manila. http://dx.doi. org/10.5962/bhl.title.48447

Blume C. L. 1825. Bijdragen tot de flora van Nederlandsch Indië. Lands Drukkerij, Batavia. http:// dx.doi.org/10.5962/bhl.title.395

Blume C.L. 1830. Flora javae (Anonaceae). J. Frank, Brussels. http://dx.doi.org/10.5962/bhl.title.48445

Boerlage J.G. 1899a. Notes sur les Anonacées du jardin botanique de Buitenzorg. Icones Bogorienses 1 (2-3): 79-208.

Boerlage J.G. 1899b. Catalogus Plantarum Phanerogamarum quae in Horto Botanico Bogoriensi Coluntur Herbaceis Exceptis. Fascicle 1. Lands Drukkerij, Batavia.

Bunchalee P. \& Chantaranothai P. 2006. Notes on Polyalthia (Annonaceae). Thai Forest Bulletin (Botany) 34: 1-3.

Candolle A.P. de 1828. Prodromus systematis naturalis regni vegetabilis. Volume 3. Treuttel \& Würtz, Paris. http://dx.doi.org/10.5962/bhl.title.286

Chaowasku T., Johnson D.M., van der Ham R.W.J.M. \& Chatrou L.W. 2012. Characterization of Hubera (Annonaceae), a new genus segregated from Polyalthia and allied to Miliusa. Phytotaxa 69: 33-56. 
Chaowasku T., Johnson D.M., van der Ham R.W.J.M. \& Chatrou L.W. 2015. Huberantha, a replacement name for Hubera (Annonaceae: Malmeoideae: Miliusae). Kew Bulletin 70 (2)-23: 1-4. http://dx.doi. org/10.1007/s12225-015-9571-Z

Craib W.G. 1924. Contributions to the flora of Siam. Additamentum XIV. Bulletin of Miscellaneous Information, Kew 1924: 81-98. http://dx.doi.org/10.2307/4111779

Craib W.G. 1925. Contributions to the flora of Siam. Additamentum XV. Bulletin of Miscellaneous Information, Kew 1925: 7-23. http://dx.doi.org/10.2307/4107434

Diels L. 1912. Beiträge zur Flora von Papuasien. Serie I. 8. Die Anonaceen von Papuasien. Botanische Jahrbücher für Systematik, Pflanzengeschichte und Pflanzengeographie 49: 113-167.

Diels L. 1931. Anonaceae novae. Notizblatt des Botanischen Gartens und Museums zu Berlin-Dahlem 11: 73-86. http://dx.doi.org/10.2307/3994585

Dunal M.F. 1817. Monographie de la famille des Anonacées. Treuttel \& Würtz, Paris.

Elmer A.D.E. 1913. New Anonaceae. Leaflets of Philippine Botany 5: 1705-1750.

Finet A. \& Gagnepain F. 1906. Contribution à la flore de l'Asie orientale. Mémoires de la Société Botanique de France 4: 55-170.

Hamidah M., Chua L.S.L., Suhaida M., Yong W.S.Y. \& Kiew R. 2011. Botanical Gazetteer for Peninsular Malaysia. Research Pamphlet no. 131. Forest Research Institute Malaysia, Kepong.

Hasskarl J.K. 1842. Plantarum genera et species novae aut reformatae javenses. Flora (Beiblätter) 252 (2): $1-56$.

Hemsley W.B. 1887. Unona wrayi. Hooker's Icones Plantarum 16: t. 1553.

Henschel A.W.E.T. 1833. Vita G. E. Rumphii. Schulzium \& Socios, Breslau.

Hooker J.D. \& Thomson T. 1855. Flora indica. Pamplin, London. http://dx.doi.org/10.5962/bhl. $\underline{\text { title. } 50109}$

Hooker J.D. 1860. XIV. Illustrations of the floras of the Malayan Archipelago and of Tropical Africa. Transactions of the Linnean Society of London 23: 155-172.http://dx.doi.org/10.1111/j.1096-3642.1860. $\underline{\text { tb00128.x }}$

Hooker J.D. \& Thomson T. 1872. Anonaceae. In: Hooker J.D. (ed.) The flora of British India 1: 45-94. L. Reeve \& Co., London.

Huber H. 1985. Annonaceae. In: Dassanayake M. D. (ed.) A revised handbook to the flora of Ceylon 5: 1-75. A.A. Balkema, Rotterdam.

Hutchinson J. 1917. Decades Kewenses. Plantarum Novarum in Herbario Horti Regii Conservatarum. Decas XC. Bulletin of Miscellaneous Information, Kew 1917: 24-30. http://dx.doi.org/10.2307/4113411

Hutchinson J. 1923. Contributions towards a phylogenetic classification of flowering plants. II. The genera of Annonaceae. Bulletin of Miscellaneous Information, Kew 1923: 241-261. http://dx.doi. org $/ 10.2307 / 4120580$

IUCN 2012. IUCN Red List Categories and Criteria: Version 3.1. Second edition. IUCN, Gland.

Johnson D.M. \& Murray N.A. 1999. Four new species of Polyalthia (Annonaceae) from Borneo and their relationship to Polyalthia insignis. Contributions from the University of Michigan Herbarium 22: 95-104.

King G. 1892. Materials for a flora of the Malay Peninsula. No. 4. Journal of the Asiatic Society of Bengal. Part II (Natural History \&c.) 61: 1-130. 
King G. 1893. The Annonaceae of British India. Annals of the Royal Botanic Garden (Calcutta) 4, Bengal Secretariat Book Depot, Calcutta.

Kochummen K.M., Ng F.S.P. \& Whitmore T.C. 1970. Notes on the systematy of Malayan phanerogams: VI-X. Federation Museums Journal 13: 133-137.

Kochummen K.M. 1972. Annonaceae. In: Whitmore T.C. (ed.) Tree Flora of Malaya 1: 61-99. Longman Malaysia Sdn Bhd, Kuala Lumpur.

Kurz W.S. 1874. Contributions towards a knowledge of the Burmese flora - Part I. Journal of the Asiatic Society of Bengal. Part II (Natural History \&c.) 43: 39-141.

Merrill E.D. 1905. Notes on Cuming's Philippine plants in the herbarium of the Bureau of Government Laboratories. Publications of the Bureau of Science Government Laboratories 35: 69-77.

Merrill E.D. 1906. New or noteworthy Philippine plants, V. Philippine Journal of Science 1, supplement III: $169-246$.

Merrill E.D. 1913. Plantae wenzelianae. Philippine Journal of Science. Section C, Botany 8: 363-390.

Merrill E.D. 1915. Studies on Philippine Annonaceae I. Philippine Journal of Science. Section C, Botany 10: 227-264.

Merrill E.D. 1921. A bibliographic enumeration of Bornean plants. Journal of the Straits Branch of the Royal Asiatic Society, special number: 1-637. http://dx.doi.org/10.5962/bhl.title.28790

Merrill E.D. 1922. New or noteworthy Bornean plants. Journal of the Straits Branch of the Royal Asiatic Society 85: 151-201.

Merrill E.D. 1923. An enumeration of Philippine flowering plants. Volume 2. Bureau of Printing, Manila. http://dx.doi.org/10.5962/bhl.title.49412

Miquel F.A.W. 1858. Flora van Nederlandsch Indië. Volume 1, part 2. C.G. van der Post, Amsterdam.

Miquel F.A.W. 1861. Flora van Nederlansch Indië. Supplementum primum. Prodromus florae sumatranae. C.G. van der Post, Amsterdam.

Miquel F.A.W. 1865. Anonaceae Archipelagi Indici. Annales Musei Botanici Lugduno-Batavi 2: 1-45.

Mols J.B., Keßler P.J.A., Rogstad S.H. \& Saunders R.M.K. 2008. Reassignment of six Polyalthia species to the new genus Maasia (Annonaceae): molecular and morphological congruence. Systematic Botany 33: 490-494. http://dx.doi.org/10.1600/036364408785679752

Mueller F.J.H. von 1877. Descriptive notes on Papuan plants. Appendix. George Skinner, Melbourne.

Okada H. 1996. New genus and new species of the Annonaceae from the Malesian wet tropics. Acta Phytotaxonomica et Geobotanica 47: 1-9.

Rauschert S. 1982. Nomina nova generica et combinationes nova spermatophytorum et pteridophytorum. Taxon 31: 554-563. http://dx.doi.org/10.2307/1220694

Ridley H.N. 1910. New or rare Malayan plants. Series V. Journal of the Straits Branch of the Royal Asiatic Society 54: 1-61.

Ridley, H.N. 1911. The flora of Lower Siam. Journal of the Straits Branch of the Royal Asiatic Society 59: 12-234.

Ridley H.N. 1912. A botanical excursion to Pulau Adang. Journal of the Straits Branch of the Royal Asiatic Society 61: 45-65.

Ridley H.N. 1917. New and rare Malayan plants. Journal of the Straits Branch of the Royal Asiatic Society 75: 5-38. 
Ridley H.N. 1920. New and rare species of Malayan plants. Journal of the Straits Branch of the Royal Asiatic Society 82: 168-204.

Ridley H.N. 1922. The flora of the Malay Peninsula. Volume 1. L. Reeve \& Co., London. http://dx.doi. org/10.5962/bhl.title.10921

Rogstad S.H. 1989. The biosystematics and evolution of the Polyalthia hypoleuca complex (Annonaceae) of Malesia, I. Systematic treatment. Journal of the Arnold Arboretum 70: 153-246.

Roxburgh W. 1795. Plants of the Coast of Coromandel. Volume 1, part 2. East India Company, London.

Ruiz H. \& Pavon J. 1798. Systema vegetabilium florae peruvianae et chilensis. De Sancha, Madrid. http://dx.doi.org/10.5962/bhl.title.887

Rumphius G.E. 1743. Herbarium amboinense. Volume 3. F. Changuion, J. Catuffe \& H. Uytwerf, Amsterdam.

Safford W.E. 1912. Desmos the proper generic name for the so-called Unonas of the Old World. Bulletin of the Torrey Botanical Club 39: 501-508. http://dx.doi.org/10.2307/2479122

Saunders R.M.K. \& Xue B. 2011. (1992) Proposal to conserve the name Enicosanthum against Monoon (Annonaceae). Taxon 60: 236-237.

Sinclair J. 1955. A revision of the Malayan Annonaceae. Gardens'Bulletin, Singapore 14: 149-516.

Sydow P. 1899. Fungi natalenses. Beiblatt zur „Hedwigia” 38: 130-134.

Thwaites G.H.K. 1864. Enumeratio plantarum zeylaniae. Part 5. Dulau \& Co., London. http://dx.doi. org/10.5962/bhl.title.574

Turner I.M. 2007. Two new names and two new combinations in Malesian Annonaceae. Gardens' Bulletin, Singapore 58: 275-278.

Turner I.M. 2009. New species and new records for Polyalthia (Annonaceae) in Borneo. Folia Malaysiana 9: 77-98.

Turner I.M. 2010a. A consideration of Cleistopetalum and a new combination in Polyalthia (Annonaceae). Phytotaxa 8: 41-45. http://dx.doi.org/10.11646/phytotaxa.8.1.4

Turner I.M. 2010b. New species of Polyalthia (Annonaceae) from Borneo and their relationship to Polyalthia cauliflora. Nordic Journal of Botany 28: 267-279. http://dx.doi.org/10.1111/j.1756$\underline{1051.2009 .00627 . \mathrm{x}}$

Turner I.M. 2011. A catalogue of the Annonaceae of Borneo. Phytotaxa 36: 1-120. http://dx.doi. org/10.11646/phytotaxa.36.1.1

Turner I.M. 2012. The plant taxa of H.N. Ridley, 4. The primitive angiosperms (Austrobaileyales, Canellales, Chloranthales, Laurales, Magnoliales, Nyphaeales and Piperales). Gardens' Bulletin, Singapore 64: 221-256.

Turner I.M. [with contributions by A.D. Weerasooriya, R.M.K. Saunders \& S.K. Ganesan] 2014. Annonaceae. In: Soepadmo E., Saw L.G., Chung R.C.K. \& Kiew R. (eds) Tree Flora of Sabah and Sarawak 8: 1-200. FRIM, Kepong.

Turner I.M. \& Utteridge T.M.A. 2015. A new species of Monoon (Annonaceae) from Peninsular Malaysia. Webbia 70: 99-102. http://dx.doi.org/10.1080/00837792.2015.1030256

Wight R. \& Arnott G.A.W. 1834. Prodromus florae peninsulae indiae orientalis. Parbury, Allen \& Co., London. http://dx.doi.org/10.5962/bhl.title.252 
Xue B., Su Y.C.F., Mols J.B., Keßler P.J.A. \& Saunders R.M.K. 2011. Further fragmentation of the polyphyletic genus Polyalthia (Annonaceae): molecular phylogenetic support for a broader delimitation of Marsypopetalum. Biodiversity and Systematics 9: 17-26. http://dx.doi.org/10.1080/14772000.2010. $\underline{542497}$

Xue B., Su Y.C.F., Thomas D.C. \& Saunders R.M.K. 2012. Pruning the polyphyletic genus Polyalthia (Annonaceae) and resurrecting the genus Monoon. Taxon 61: 1021-1039.

Zollinger H. 1858. Uber die Anonaceen des ostindischen Archipels. Linnaea 29: 297-325.

Manuscript received: 24 August 2015

Manuscript accepted: 25 November 2015

Published on: 22 March 2016

Topic editor: Koen Martens

Desk editor: Natacha Beau

Printed versions of all papers are also deposited in the libraries of the institutes that are members of the EJT consortium: Muséum national d'Histoire naturelle, Paris, France; Botanic Garden Meise, Belgium; Royal Museum for Central Africa, Tervuren, Belgium; Natural History Museum, London, United Kingdom; Royal Belgian Institute of Natural Sciences, Brussels, Belgium; Natural History Museum of Denmark, Copenhagen, Denmark. 